\title{
Likelihood of being Physically Inactive from a Nationally Representative sample of Autistic Children
}

\author{
Vijay Vasudevan ${ }^{1}$ \\ 1. Autism Speaks, Princeton, NJ
}

Corresponding Author: Vijay Vasudevan, $\mathrm{PhD}$

Autism Speaks

1060 State Road, $2^{\text {nd }}$ Floor

Princeton, NJ 08540

vijay.vasudevan@autismspeaks.org

(609)-968-6615

Running Title: Physical Inactivity among Autistic Children

Conflict of Interest: The authors have no conflicts of interest to declare.

Grant Sponsor: This research did not receive any specific grant from funding agencies in the public, commercial, or not-for-profit sectors.

Grant Number: N/A

Number of Text Pages: 13

Number of Tables: 3

Number of Figures: 0 


\begin{abstract}
Despite the many health risks of physical inactivity, studies have demonstrated individual, family, and environmental determinants of inactivity for autistic children. However, these studies never examined these correlates at the same time. Therefore, the purpose of this study was to explore these ecological domains concurrently when examining physical inactivity correlates for autistic children. This study used data from the 2016-2020 National Survey of Children's Health. The authors predicted physical inactivity while controlling for child, parental/household, and neighborhood correlates with autism status as the comparison group. When controlling for covariates, children with co-occurring autism and intellectual and developmental disability (IDD) (adjusted odds ratio $(\mathrm{aOR})=1.91,95 \%$ confidence interval $(\mathrm{CI})$ : 1.36-2.68) or ASD only ( $\mathrm{aOR}=1.91, \mathrm{CI}: 1.48-2.48)$ were significantly more likely to be inactive when compared to children without autism or IDD. However, autism medicine and autism severity were not predictors for obese autistic children. These findings indicate that it is important to take a holistic, ecological approach when exploring the correlates of inactivity for autistic children.
\end{abstract}

Keywords: Autism Spectrum Disorders (ASD), Physical Inactivity, Obesity 


\section{INTRODUCTION}

The health risks of being overweight and obese are well known including: type 2 diabetes, high blood pressure, coronary heart disease, sleep apnea and breathing problems, depression, anxiety, and body pain. ${ }^{1}$ Autistic children and adolescents are at greater risk of being overweight/obese and having unmet healthcare needs. ${ }^{2,3}$ Some of the contributing factors to being obese for the autistic children include physical inactivity, sleep quality, and parental concern about weight. ${ }^{4-6}$ McCoy (2020) found an inverse relationship between being active seven days a week and obesity status of autistic children. ${ }^{7}$

Physical activity has been shown to improve weight status while also addressing the health risks of being obese, such as improved cardiorespiratory health and muscular fitness, improved cardiometabolic health, and reduced risk of depression. ${ }^{8}$ To reach these benefits, all children and youth are recommended to get at least 60 minutes of moderate to vigorous physical activity every day. ${ }^{8}$ Despite these benefits, approximately a quarter of all children age six to 17 met this guideline. ${ }^{9}$ Children and adolescents with disabilities had significantly lower odds of meeting the physical activity guidelines. ${ }^{10}$ Barriers to physical activity for people with disabilities exist at multiple domains of the ecological model, including individual factors like health, family perception, and having a supportive environment. ${ }^{11,12}$

A previous study, by Case (2020) which explored the likelihood of meeting the physical activity guidelines, found that children with an autism diagnosis were $35 \%$ less likely to meet the physical activity guidelines when compared to children without an autism diagnosis. However, in an adjusted model for autism severity, having multiple developmental disabilities, and having a health condition that limits the child's ability to do activities, autism was not a significant predictor of meeting the physical activity guideline. ${ }^{13}$ This study did not control for important covariates such as sleep, obesity status, parental concern about weight, and living in a supportive neighborhood. Therefore, the purpose of this study was to explore the likelihood to meet the physical activity guidelines by autism status while controlling for ecological correlates. 


\section{METHODS}

\section{$\underline{\text { Design }}$}

This study used combined data from the 2016-2020 National Survey of Children's Health (NSCH). The NSCH is a nationally, representative sample survey which is administered annually by the US Census Bureau for the Maternal Child Health Bureau, Health Resources and Services Administration. The NSCH asks age-based topical questions (0-5 years, 6-11 years, and 12-17 years old). All responses are parent-reported. Individual survey weights were divided by the number combined survey years in order to derive nationally, representative estimates. Because NSCH data were publicly available, the authors did not seek Institutional Review Board approval.

\section{$\underline{\text { Autism Status }}$}

Because a third of all children with ASD had an intellectual disability (IDD), ${ }^{14}$ one mutually exclusive variable for autism status was created. which had the following responses: no autism and IDD, autism only, and autism with co-occurring IDD. The NSCH asks two questions to assess current status of ASD and IDD. Current autism status was determined by two questions: (1) "Has a doctor or other health care provider EVER told you that this child has Autism or Autism Spectrum Disorder (ASD)? Include diagnoses of Asperger's Disorder or Pervasive Developmental Disorder (PDD)." and (2) "If yes, does this child CURRENTLY have the condition?" Children whose parents who reported "yes" to both items were categorized with being "Currently diagnosed with autism." If the parent reported "no" to either question, the child was categorized as "Not currently diagnosed with autism." Current IDD status was determined by two questions: (1) "Has a doctor, other health care provider, or educator EVER told you that this child has... Intellectual Disability (formerly known as Mental Retardation)? Examples of educators are teachers and school nurses." and (2) "If yes, does this child CURRENTLY have the condition?" Children whose parents who reported "yes" to both items were categorized with being "Currently diagnosed with IDD." If the parent reported "no" to either question, the child was categorized as "Not currently diagnosed with IDD."

Parents who reported that their child are currently diagnosed with ASD were also asked for their child's autism severity (rated as mild, moderate, or severe) and if the child is taking medicine for autism. 


\section{Physical Activity}

Physical activity was asked of children who were from six to 17 years old. Physical activity was asked by the NSCH with one item: "DURING THE PAST WEEK, on how many days did this child exercise, play a sport, or participate in physical activity for at least 60 minutes?" Response options included: 0 days, 1-3 days, 4-6 days, and every day. The authors collapsed the responses into three categories: Inactive -0 days, Insufficiently active -1 to 6 days, and Active - 7 days. A second dichotomous variable was created: Inactive versus Active or Insufficiently Active.

\section{$\underline{\text { Correlates }}$}

The authors controlled for child, parent/household, and community variables. Child variables include age (6-11 years old and 12-17 years old), gender, race (White, non-Hispanic, Black, non-Hispanic, Hispanic, and Multirace or Other, non-Hispanic), obesity category (underweight, normal weight, overweight, and obese), and sleep. Parent/household variables include parent's highest education, parent concern about child's weight, and federal poverty level (FPL). The neighborhood variable was whether or not the child lives in a supportive neighborhood.

\section{$\underline{\text { Analyses }}$}

Stata version 16.1 was used for all analyses. ${ }^{15}$ Weighted, demographic prevalence estimates (\%) and 95\% confidence intervals (CIs) were calculated for survey respondents of all respondents whose child was at least six years old. Chi-square analysis was performed to identify differences in physical activity level (inactive, insufficiently active, and active) and autism status. Unadjusted and adjusted odds ratios (aOR) were calculated, using logistic regression, to predict physical inactivity (inactive versus active or insufficiently active) controlling for child, parental/household, and neighborhood correlates with autism status as the comparison group.

A separate logistic regression, of just autistic children who were obese, was performed to predict physical inactivity, while controlling for the same correlates stated above, and adding autism severity and whether or not the autistic child is taking medicine to treat their autism. 


\section{RESULTS}

Table 1 presents the prevalence estimates and CIs for the demographic variables of all survey participants, whose children were at least six years old. Approximately three percent of children were currently diagnosed with ASD. Roughly a third (34.8\%) of parents reported that their children did not get the recommended hours of sleep. About $15 \%$ of parents reported that their children were overweight or obese (15.6\% and $15.8 \%$ respectively), and $7.6 \%$ was concerned that their child's weight was too high. Finally, $10.1 \%$ of parents reported that their child got zero days of at least 60 minutes of activity, while just $22.1 \%$ were active every day.

\section{INSERT TABLE 1 HERE}

Children with co-occurring ASD and IDD or ASD only were statistically more likely to be "Inactive - 0 days" when compared to children who do not have ASD $(21.0 \%$ and $17.3 \%$ versus $9.4 \%$ respectively, $\chi^{2}=16.4, \mathrm{p}<0.001$ ). When controlling for covariates (Table 2 ), children with co-occurring ASD and IDD (aOR = 1.91, CI: 1.36-2.68) or ASD only $(\mathrm{aOR}=1.91$, CI: 1.48-2.48) were significantly more likely to be inactive when compared to children without ASD or IDD.

\section{INSERT TABLE 2 HERE}

When exploring autistic children who were obese (Table 3), the significant correlates of physical inactivity were parent concern that their child's weight is too high (aOR $=02.97, \mathrm{CI}$ : 1.28-6.87) and the child's age is between 12 and 17 years old (aOR = 5.18, CI: 1.63-16.51). Parent-rated severity of their child's autism and whether or not their autistic child is taking medicine for their autism were not significant predictors.

\section{INSERT TABLE 3 HERE}




\section{DISCUSSION}

Previous studies on correlates of physical activity for autistic children focused only on just one aspect of the child's life, such as the individual/parental/community influences. This study builds upon previous research into exploring physical activity promotion for autistic children, by using an ecological approach.

Because physical activity, obesity, and autism are interwoven, programs that promote physical activity for autistic children should be flexible to meet the needs of the individual child. In a meta-analysis of physical activity interventions for autistic children, despite the effect size, physical activity interventions had low impact for social functioning or body composition. ${ }^{16,17}$ While current physical activity interventions have effects on muscular strength and endurance, locomotor, manipulative skills, and skill-related fitness, they did not assess physical activities' holistic impact on the health and well-being of the autistic child, such as improved sleep and weight. In this study, a lack of sleep was associated an aOR of 1.16 for physical inactivity (CI: 1.04-1.30). Additionally, the previous interventions identified in the metanalysis do not utilize efforts like social connectedness efforts like peer-promoters or caregivers to increase physical activity. The study findings showed that when parent concern about their child's weight was high, their child was more likely to be inactive (aOR $=1.89, \mathrm{CI}$ : $1.59-2.25)$. Physical activity programs for people with IDD have been successful when incorporating parent caregivers and peer promoters. ${ }^{18-20}$ Future interventions could utilize technology like Fitbit activity watches and scales to track the intervention's impact on the autistic child's (with or without co-occurring IDD) sleep, physical activity, and weight by facilitating caregiver and peer involvement.

Additionally, physical education environments had the highest effect size in promoting physical activity. ${ }^{16}$ This finding emphasizes the importance of creating inclusive physical activity environments for autistic children. Inclusive environments allow everyone to take full part in the same program and services by reducing barriers and improving the physical/social well-being. ${ }^{21}$ The Special Olympics and National Center on Health, Physical Activity, and Disability (NCHPAD) have created efforts to promote inclusive physical activity environments, especially at school settings. ${ }^{21,22}$ For example, NCHPAD has a pilot micro-grant to local communities to establish a disability inclusion program to promotion health, physical activity, and weight 
management. ${ }^{23}$ Disability, physical activity, and governmental organizations could promote ASD inclusion in physical activity programs and activities.

This study is subject to three limitations. First, data from the NSCH is limited to selfreported data. Parents are somewhat likely to provide socially desirable responses for weight and physical activity. This might lead to an underreporting of weight and an overreporting of physical activity. However, the authors expect the social desirability of responses to be the same by ASD/IDD status and thus the aORs would be conservative point estimates. Secondly, the authors cannot determine causality because data from the NSCH are cross-sectional. Finally, interpretations from the study are limited to the variables that are available in the publicly available data set. For example, while the NSCH provides a measure of whether or not the child lives in a metropolitan statistical area, the NSCH does not measure urban versus rural. This is important because these are two different metrics for how the federal government measures density of a community. ${ }^{24,25}$

Having a child with autism, especially with cooccurring IDD, has been associated with many negative health behaviors including physical inactivity and health risks like obesity. Having knowledge about the correlates between autism status and physical inactivity can help organizations, like schools, tailor their programs to be inclusive to autistic children. 


\section{REFERENCES}

1. Centers for Disease Control and Prevention, Division of Nutrition, Physical Activity, and Obesity, National Center for Chronic Disease Prevention and Health Promotion. The Health Effects of Overweight and Obesity. 2020; https://www.cdc.gov/healthyweight/effects/index.html. Accessed October 20, 2021.

2. Hill AP, Zuckerman KE, Fombonne E. Obesity and Autism. Pediatrics. 2015;136(6):1051-1061.

3. Karpur A, Lello A, Frazier T, Dixon PJ, Shih AJ. Health Disparities among Children with Autism Spectrum Disorders: Analysis of the National Survey of Children's Health 2016. J Autism Dev Disord. 2019;49(4):1652-1664.

4. Tybor DJ, Eliasziw M, Kral TVE, et al. Parental concern regarding obesity in children with autism spectrum disorder in the United States: National Survey of Children's Health 2016. Disability and Health Journal. 2019;12(1):126-130.

5. Curtin C, Anderson SE, Must A, Bandini L. The prevalence of obesity in children with autism: a secondary data analysis using nationally representative data from the National Survey of Children's Health. BMC Pediatrics. 2010;10(1):11.

6. Buro AW, Salinas-Miranda A, Marshall J, Gray HL, Kirby RS. Correlates of obesity in adolescents with and without autism spectrum disorder: The 2017-2018 National Survey of Children's Health. Disabil Health J. 2021:101221.

7. McCoy SM, Morgan K. Obesity, physical activity, and sedentary behaviors in adolescents with autism spectrum disorder compared with typically developing peers. Autism. 2020;24(2):387-399.

8. U.S. Department of Health and Human Services. Physical Activity Guidelines for Americans. 2nd edition. Washington DC2018.

9. Centers for Disease Control and Prevention. Physical Activity Facts. 2020; https://www.cdc.gov/healthyschools/physicalactivity/facts.htm. Accessed October 15, 2021.

10. Ross SM, Smit E, Yun J, Bogart K, Hatfield B, Logan SW. Updated National Estimates of Disparities in Physical Activity and Sports Participation Experienced by Children and Adolescents With Disabilities: NSCH 2016-2017. Journal of Physical Activity and Health. 2020;17(4):443-455.

11. Vasudevan V, Rimmer JH, Kviz F. Development of the Barriers to Physical Activity Questionnaire for People with Mobility Impairments. Disabil Health J. 2015;8(4):547556.

12. Must A, Phillips S, Curtin C, Bandini LG. Barriers to Physical Activity in Children With Autism Spectrum Disorders: Relationship to Physical Activity and Screen Time. Journal of Physical Activity and Health. 2015;12(4):529-534.

13. Case L, Ross S, Yun J. Physical activity guideline compliance among a national sample of children with various developmental disabilities. Disabil Health J. 2020;13(2):100881.

14. Maenner MJ, Shaw KA, Baio J, et al. Prevalence of Autism Spectrum Disorder Among Children Aged 8 Years - Autism and Developmental Disabilities Monitoring Network, 11 Sites, United States, 2016. Morbidity and mortality weekly report Surveillance summaries (Washington, DC : 2002). 2020;69(4):1-12.

15. Stata Statistical Software: Release 16 [computer program]. College Station, TX: StataCorp LLC; 2019. 
16. Healy S, Nacario A, Braithwaite RE, Hopper C. The effect of physical activity interventions on youth with autism spectrum disorder: A meta $\square$ analysis. Autism Research. 2018;11(6):818-833.

17. Reinders NJ, Branco A, Wright K, Fletcher PC, Bryden PJ. Scoping Review: Physical Activity and Social Functioning in Young People With Autism Spectrum Disorder. Frontiers in Psychology. 2019;10(120).

18. Marks B, Sisirak J, Magallanes R, Krok K, Donohue-Chase D. Effectiveness of a HealthMessages Peer-to-Peer Program for People With Intellectual and Developmental Disabilities. Intellectual and developmental disabilities. 2019;57(3):242-258.

19. Temple VA, Frey GC, Stanish HI. Interventions to promote physical activity for adults with intellectual disabilities. Salud Pública de México. 2017;59(4):446-453.

20. Hsieh K. We Walk: A pilot technology intervention to promote physical activity for adults with ID and their caregivers. In. Vol \$20,000. Chicago, IL: College of Applied Health Sciences Interdisciplinary Pilot Grant; 2016.

21. Special Olympics. Inclusive Health. 2021; https://www.specialolympics.org/ourwork/inclusive-health. Accessed November 1, 2021.

22. National Center on Health PAaD. Inclusive Health Coalition Initiative. 2021; https://www.nchpad.org/1149/5665/Inclusive $\sim$ Health Coalition Initiative. Accessed November 1, 2021.

23. National Center on Health, Physical Activity and Disability,. Accelerating Disability Inclusion through Community-Based Microgrants (During COVID-19). 2021; https://www.nchpad.org/1784/6958/Accelerating Disability Inclusion through Commu nity-Based Microgrants During COVID-19 . Accessed November 03, 2021.

24. Koontz LD. Geographic Information Systems: Challenges to Effective Data Sharing. Washington DC: United States General Accounting Office;2003.

25. Census Bureau. Urban Areas for the 2020 Census-Proposed Criteria. In: Bureau of the Census, Department of Commerce., ed. Washington DC2021. 


\section{Table 1: Demographics}

\section{Current Autism Status}

Not currently diagnosed with autism

Prevalence Estimate

$95 \% \mathrm{CI}$

Currently have autism

$96.9 \%$

$(96.7 \%, 97.1 \%)$

Child age

6-11 years old

$3.1 \%$

$(2.9 \%, 3.3 \%)$

12-17 years old

$15.6 \%$

$(15.2 \%, 16.1 \%)$

Gender

Female

$84.4 \%$

$(84.0 \%, 84.9 \%)$

Male

$48.9 \%$

$(48.4 \%, 49.4 \%)$

Race

White, non-Hispanic

$51.1 \%$

$(50.6 \%, 51.7 \%)$

Black, non-Hispanic

$50.7 \%$

$(50.2 \%, 51.3 \%)$

Hispanic

$13.2 \%$

$(12.8 \%, 13.6 \%)$

Multirace or Other, non-Hispanic

$25.2 \%$

$(24.6 \%, 25.8 \%)$

Adequate amount of age

Child sleeps recommended age-appropriate hours

$10.9 \%$

$(10.6 \%, 11.2 \%)$

Child sleep less than recommended age-appropriate hours

$65.3 \%$

$(64.7 \%, 65.8 \%)$

Parent highest education

College degree or higher

$34.8 \%$

$(34.2 \%, 35.3 \%)$

Some college or associate degree

$49.6 \%$

$(49.0 \%, 50.1 \%)$

High school graduate or less

$21.8 \%$

$(21.4 \%, 22.2 \%)$

Federal Poverty Level

Greater than or equal to $200 \%$ FPL

$28.6 \%$

$(28.1 \%, 29.2 \%)$

Less than 200\% FPL

$58.6 \%$

$(58.0 \%, 59.1 \%)$

Does child live in a supportive neighborhood

Do not live-in supportive neighborhood

$41.4 \%$

$(40.9 \%, 42.0 \%)$

Live in supportive neighborhood

$45.5 \%$

$(44.9 \%, 44.6 \%)$

Parent-reported Child Weight Category

Underweight (less than 5th percentile)

Normal weight ( 5 th to 84 th percentile)

$54.5 \%$

$(54.0 \%, 55.1 \%)$

Overweight (85th to 94 th percentile)

$6.5 \%$

$(6.1 \%, 6.9 \%)$

Obese (95th percentile or above)

$62.1 \%$

$(61.3 \%, 62.9 \%)$

$15.6 \%$

$(15.0 \%, 16.2 \%)$

Parent concerned about child's weight

No, not concerned

$15.8 \%$

$(15.2 \%, 16.5 \%)$

Yes, concerned it is too high

$89.4 \%$

$(89.1 \%, 89.8 \%)$

Yes, concerned it is too low

$7.6 \%$

$(7.4 \%, 8.0 \%)$

$2.9 \%$

$(2.8 \%, 3.1 \%)$

Physical activity at least 60 minutes per day

Inactive - 0 days

$10.1 \%$

$(9.7 \%, 10.5 \%)$

Insufficiently active - 1-6 days

$67.8 \%$

$(67.2 \%, 68.4 \%)$

Active - 7 days

$22.1 \%$

$(21.6 \%, 22.7 \%)$ 
medRxiv preprint doi: https://doi.org/10.1101/2021.11.05.21265973; this version posted November 9, 2021. The copyright holder for this preprint (which was not certified by peer review) is the author/funder, who has granted medRxiv a license to display the preprint in perpetuity.

It is made available under a CC-BY-NC-ND 4.0 International license.

Table 2: Likelihood of Being Inactive

\begin{tabular}{|c|c|c|c|c|}
\hline & Unadjusted Odds Ratio & dds Ratio & \multicolumn{2}{|c|}{ Adjusted Odds Ratio } \\
\hline & Estimate & $95 \% \mathrm{CI}$ & Estimate & $95 \% \mathrm{CI}$ \\
\hline \multicolumn{5}{|l|}{ Current Autism Status } \\
\hline $\begin{array}{l}\text { Not currently diagnosed with autism } \\
\text { or intellectual/developmental disability }\end{array}$ & REF & & REF & \\
\hline Currently have autism only & 2.01 & $(1.49,2.70)$ & 1.91 & $(1.36,2.68)$ \\
\hline Currently have autism and IDD & 2.56 & $(1.88,3.48)$ & 1.91 & $(1.48,2.48)$ \\
\hline \multicolumn{5}{|l|}{ Child age } \\
\hline 6-11 years old & & & REF & \\
\hline 12-17 years old & & & 2.27 & $(1.73,1.68)$ \\
\hline \multicolumn{5}{|l|}{ Gender } \\
\hline Male & & & REF & \\
\hline Female & & & 1.51 & $(1.35,1.68)$ \\
\hline \multicolumn{5}{|l|}{ Race } \\
\hline White, non-Hispanic & & & REF & \\
\hline Black, non-Hispanic & & & 1.52 & $(1.31,1.76)$ \\
\hline Hispanic & & & 1.04 & $(0.90,1.20)$ \\
\hline Multirace or Other, non-Hispanic & & & 1.32 & $(1.12,1.54)$ \\
\hline \multicolumn{5}{|l|}{ Parent highest education } \\
\hline College degree or higher & & & REF & \\
\hline Some college or associate degree & & & 1.27 & $(1.13,1.43)$ \\
\hline High school graduate or less & & & 1.61 & $(1.40,1.84)$ \\
\hline \multicolumn{5}{|l|}{ Federal Poverty Level } \\
\hline Greater than or equal to $200 \% \mathrm{FPL}$ & & & REF & \\
\hline Less than $200 \%$ FPL & & & 1.07 & $(0.94,1.21)$ \\
\hline \multicolumn{5}{|l|}{ Does child live in a supportive neighborhood } \\
\hline Live in supportive neighborhood & & & REF & \\
\hline Do not live in supportive neighborhood & & & 1.52 & $(1.36,1.70)$ \\
\hline \multicolumn{5}{|l|}{ Parent-reported Child Weight Category } \\
\hline Normal weight (5th to 84th percentile) & & & REF & \\
\hline Underweight (less than 5th percentile) & & & 1.23 & $(0.95,1.59)$ \\
\hline Overweight (85th to 94 th percentile) & & & 1.08 & $(0.94,1.26)$ \\
\hline Obese (95th percentile or above) & & & 1.21 & $(1.01,1.47)$ \\
\hline \multicolumn{5}{|l|}{ Parent concerned about child's weight } \\
\hline No, not concerned & & & $\mathrm{REF}$ & \\
\hline Yes, concerned it is too high & & & 1.89 & $(1.59,2.25)$ \\
\hline Yes, concerned it is too low & & & 1.67 & $(1.29,2.18)$ \\
\hline \multicolumn{5}{|l|}{ Adequate amount of age-appropriate sleep } \\
\hline Child sleeps recommended age-appropriate & & & REF & \\
\hline Child sleep less than recommended age-a & propriate ho & & 1.16 & $(1.04,1.30)$ \\
\hline
\end{tabular}


Table 2: Likelihood of Being Inactive for Autistic Children who are Obese

Adjusted Odds Ratio

Prevalence Estimate

$95 \% \mathrm{CI}$

Child age

6-11 years old

REF

12-17 years old

5.18

$(1.63,16.51)$

Gender

Male

REF

Female

0.40

$(0.17,0.93)$

Race

White, non-Hispanic

REF

Black, non-Hispanic

0.13

$(\mathbf{0 . 0 3}, 0.49)$

Hispanic

1.32

$(0.49,3.60)$

Multirace or Other, non-Hispanic

0.76

$(0.24,2.43)$

Parent highest education

College degree or higher

REF

Some college or associate degree $\quad \mathbf{0 . 4 6}$

$(0.21,0.99)$

High school graduate or less

0.46

$(0.19,1.09)$

Federal Poverty Level

Greater than or equal to $200 \%$ FPL

REF

Less than 200\% FPL

1.12

$(0.56,2.21)$

Does child live in a supportive neighborhood

Live in supportive neighborhood

REF

Do not live in supportive neighborhood

0.70

$(0.37,1.34)$

Parent concerned about child's weight

No, not concerned

REF

Yes, concerned it is too high

2.97

$(1.28,6.87)$

Adequate amount of age-appropriate sleep

Child sleeps recommended age-appropriate hours

REF

Child sleep less than recommended age-appropriate hours

1.48

$(0.76,2.89)$

Child taking medicine for autism

Not taking autism medication

REF

Taking autism medication

1.24

$(0.60,2.60)$

Parent-rated autism severity

Mild

REF

Moderate

1.18

Severe

0.51

$(0.59,2.38)$

$(0.18,1.45)$ 